JOURNAL OF

SYNCHROTRON

RADIATION

ISSN 1600-5775

Received 27 June 2019

Accepted 7 October 2019

Edited by A. F. Craievich, University of São Paulo, Brazil

Keywords: optical-trap sample holders; X-ray diffraction; nanometre-sized particles; crystallite size.

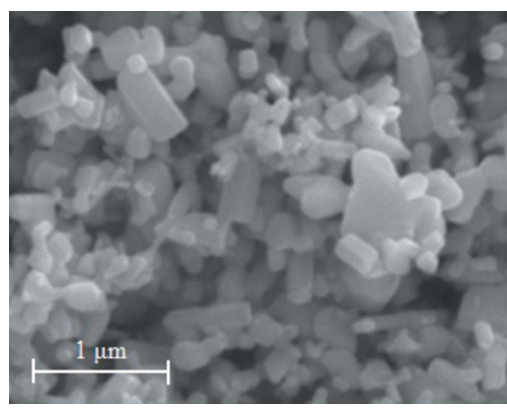

(C) 2020 International Union of Crystallography

\section{X-ray diffraction measurement of a single nanometre-sized particle levitated in air by an optical-trap sample holder}

\author{
Yoshimitsu Fukuyama, ${ }^{a *}$ Nobuhiro Yasuda, ${ }^{a}$ Kunihisa Sugimoto ${ }^{\mathrm{a}, \mathrm{b}}$ and \\ Shigeru Kimura ${ }^{a}$
}

\begin{abstract}
${ }^{a}$ Center for Synchrotron Radiation Research, Japan Synchrotron Radiation Research Institute (JASRI), 1-1-1 Kouto, Sayocho, Sayo-gun, Hyogo 679-5198, Japan, and ${ }^{\mathbf{b}}$ Institute for Integrated Cell-Material Sciences (iCeMS), Kyoto University, Yoshida-Ushinomiya-cho, Sakyo-ku, Kyoto 606-8501, Japan. *Correspondence e-mail: yfukuya@spring8.or.jp
\end{abstract}

A single-beam optical-trap sample holder for X-ray diffraction measurements with synchrotron radiation has been developed. The sample holder was used to obtain an X-ray diffraction image of a single $\mathrm{ZnO}$ particle levitated in air, without mechanical contact, by the optical gradient force exerted by a focused laser beam. The diffraction image showed a Debye ring pattern, which was similar to a powder diffraction pattern of an assemblage of $\mathrm{ZnO}$ particles. While the $\mathrm{ZnO}$ particle is held by the optical trap in air, it rotates irregularly. Therefore, the Debye ring pattern of the $\mathrm{ZnO}$ particle can be clearly obtained even if the $\mathrm{ZnO}$ particle is a single grain. Lattice parameters and crystallite size of the single $\mathrm{ZnO}$ particle were determined simultaneously. The lattice parameters were determined to be $a=3.2505 \pm 0.0005 \AA$ and $c=5.207 \pm$ $0.006 \AA$, which are consistent with those of the assemblage of $\mathrm{ZnO}$ particles. The crystallite size determined by the Scherrer method was $193.4 \pm 26.2 \mathrm{~nm}$.

\section{Introduction}

Nanometre-sized particles have been extensively investigated from both fundamental and technological viewpoints. Of particular interest is the relationship between size-dependent structures and properties. Surface effects associated with nanometre-sized particles are enhanced because the ratio of surface area to volume increases as particle size decreases. Enhancement of these effects changes the crystal structure, lattice parameters, and physical and chemical properties of the nanometre-sized particle. One of the most promising examples is barium titanate $\left(\mathrm{BaTiO}_{3}\right)$. Assemblages of nanometre-sized $\mathrm{BaTiO}_{3}$ particles packed in a glass capillary have been studied by powder diffraction. According to these studies, the structural phase changes from tetragonal to cubic as particle size decreases (Aoyagi et al., 2002; Hoshina, 2013). Unfortunately, structural parameters obtained by powder diffraction are averaged values for the whole particle assemblage, which has a certain particle-size distribution. To investigate the properties of a nanometre-sized particle, it is important to determine the crystal structure and crystallite size of a single particle simultaneously. X-ray diffraction is a powerful tool for determining not only the crystal structure but also the crystallite size with accuracy.

In general, it is difficult to obtain X-ray diffraction images of a single particle of micrometre size or less due to two problems. One is an insufficient photon flux density of monochromatic X-rays. This has recently been solved by combining a focusing technique and monochromatic X-ray beam, i.e. a synchrotron radiation beam with high brilliance 
and small divergence with a high photon flux (Riekel et al., 2005; Volkringer et al., 2007; Yasuda et al., 2009). The other problem is the absence of a method for holding a single particle as a sample. For a typical sample-holding method, a small single particle is usually attached to the top of a fine glass fibre with adhesive. However, there are three problems with this method: (i) difficulty in holding a single particle with a size of $1 \mu \mathrm{m}$ or less, (ii) lattice strain due to mechanical contact with the glass fibre and adhesive, and (iii) degradation of the signal-to-noise ratio due to undesired scattering from the glass fibre and adhesive. Unlike the first problem, the second, i.e. the absence of a sample-holding method, has not been solved until quite recently.

Radiation pressure exerted by a strongly focused laser beam can be used to levitate and manipulate a micrometresized neutral dielectric particle (Ashkin, 1970). On the basis of this manipulation principle, a single protein particle was trapped and manipulated by optical tweezers in an aqueous environment. The trapped particle, a few micrometres up to a few tens of micrometres in size, was experimentally subjected to wide-angle and small-angle X-ray scattering by combining the optical tweezers with focused synchrotron radiation. These experiments successfully demonstrated tomographic measurements on a single particle (Santucci et al., 2011). We previously developed an 'optical-trap sample holder' for suspending a single particle without mechanical contact in an air environment (Fukuyama et al., 2013). This sample holder was composed of counterpropagating laser beams. An X-ray diffraction image of a single $380 \mathrm{~nm}$ diameter particle of cerium oxide $\left(\mathrm{CeO}_{2}\right)$, held with the sample holder, was acquired by using a high-flux focused synchrotron radiation beam.

X-ray diffraction measurements of a single nanometre-sized particle have been enabled by combining the optical-trap sample holder with focused synchrotron radiation. However, as for the above-mentioned optical-trap sample holder with counterpropagating laser beams, it is difficult to align the counterpropagating laser beams on identical axes within an accuracy of $1 \mu \mathrm{m}$. Any misalignment causes instability of the trap position, and in the worst case a particle cannot be trapped. The instability of the trap position causes a decrease in the actual exposure time of the focused synchrotron radiation because the requirement of overlap between the trap position and the focused synchrotron radiation is severe. A diffraction signal from a single nanometre-sized particle is extremely weak because the number of diffracted X-rays is proportional to the volume of the sample. Insufficient actual exposure time resulting from the instability of the trap position is thus a serious problem.

To overcome this problem, we developed a new sample holder that uses a single-beam optical trap (Ashkin et al., 1986). Since the optical configuration of a single-beam optical trap is simple, the stability of the position at which the sample is trapped can be improved significantly, and that improved stability secures sufficient actual exposure time of focused synchrotron radiation for a single nanometre-sized particle. As a result, diffraction data can be reliably collected. Suffi- cient actual exposure time ensures reliability of the diffraction profile. It is well known that crystallite size can be estimated from the broadening of an X-ray diffraction profile (Scherrer, 1918; Langford \& Wilson, 1978). It is thus expected that the crystallite size of a single particle can be estimated from a reliable diffraction profile.

The ultimate aim of our study is to clarify the relationship between crystallite size and size-dependent physical properties of a single nanometre-sized particle. An optical trap in an air environment is a useful sample-holding method which facilitates determination of crystallite size and structure simultaneously without mechanical contact. In this study, a single-beam optical-trap sample holder for a single nanometre-sized particle was developed. An X-ray diffraction image of a single $\mathrm{ZnO}$ particle held in the single-beam optical trap was obtained. The crystal structure and crystallite size of the single $\mathrm{ZnO}$ particle were determined from the image obtained. The quality of the X-ray diffraction image of the single $\mathrm{ZnO}$ particle was evaluated and compared with that of an $\mathrm{X}$-ray diffraction image of an assemblage of $\mathrm{ZnO}$ particles.

\section{Instrumentation}

The system developed for X-ray diffraction measurement of a single particle was installed in an experimental hutch of the BL40XU beamline at SPring-8 (Inoue et al., 2001; Kimura et al., 2007). The system comprises a single-beam optical-trap sample holder and an X-ray diffractometer. A schematic diagram and photograph of the experimental setup of the system are shown in Figs. 1 and 2(a), respectively.

\subsection{Single-beam optical-trap sample holder}

The developed sample holder uses a single-beam optical trap. A TEM ${ }_{00}$-mode Gaussian beam emitted from an optically pumped semiconductor (OPS) laser (COHERENT Genesis MX, $\lambda=532 \mathrm{~nm}$, continuous wave) is focused with a nonspherical lens with numerical aperture of 0.5 and focal length

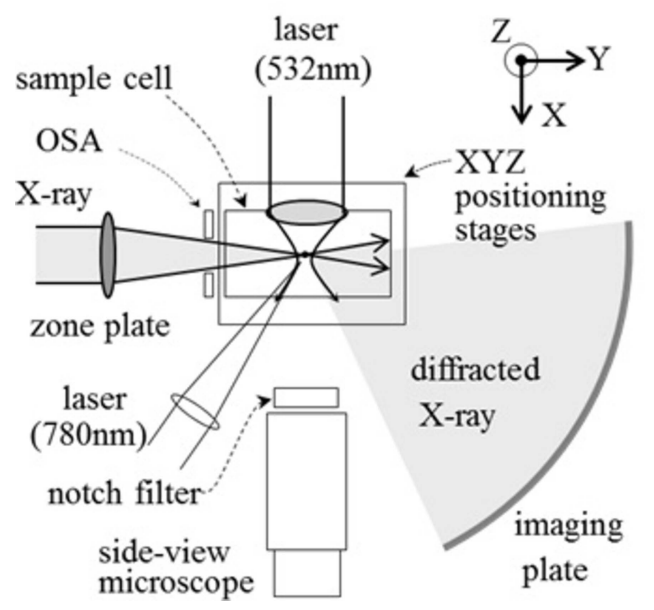

Figure 1

Experimental setup of the single-beam optical-trap sample holder and the $\mathrm{X}$-ray diffractometer (top view). For clarity, the top-view microscope (installed above the sample cell and parallel to the $z$ axis) is not shown. 

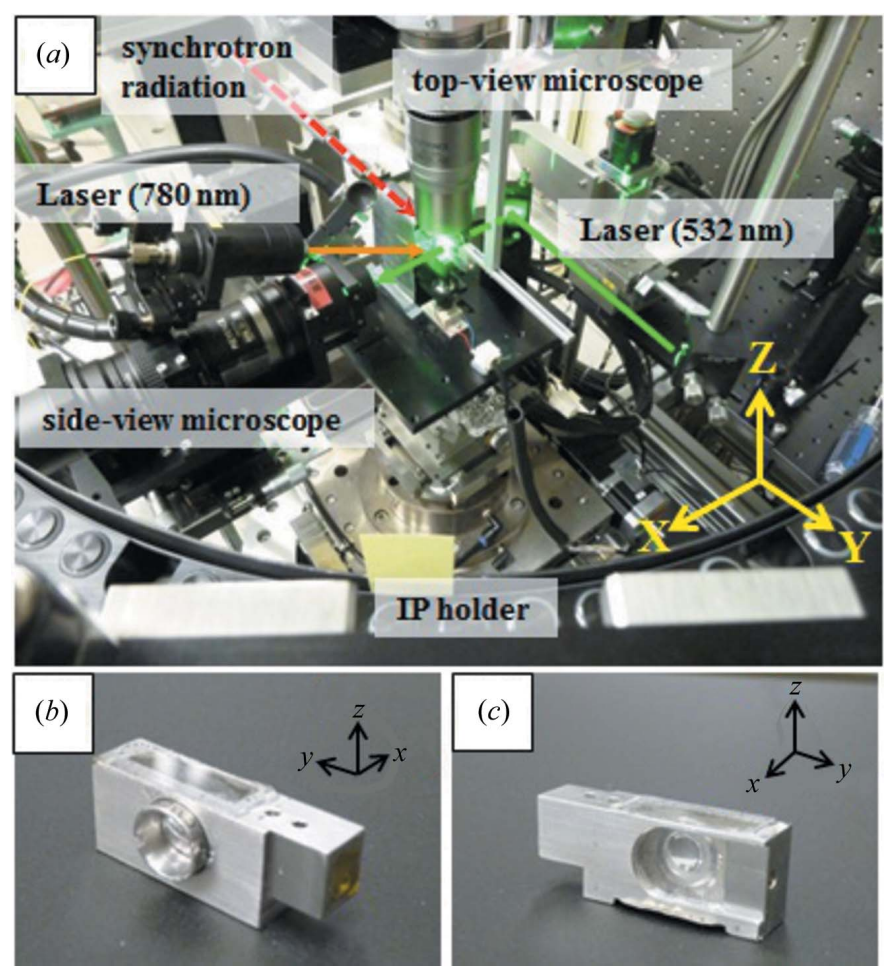

Figure 2

(a) Photograph of the single-beam optical-trap sample holder and X-ray diffractometer. The red and green arrows indicate the synchrotron radiation beam and the OPS laser beam used for the single-beam optical trap, respectively. The orange arrow indicates the semiconductor laser beam for illuminating the sample particle. $(b)$ and $(c)$ Photographs of the sample cell used for the optical trap. In $(b)$, a non-spherical lens is attached to the side face of the sample cell.

of $8 \mathrm{~mm}$. The full width at half-maximum (FWHM) of the laser beam at the focal point is $3.1 \mu \mathrm{m}$. The total power of the laser at the centre of the sample holder can be adjusted up to $1 \mathrm{~W}$. A particle with a high refractive index experiences an optical gradient force and a scattering force when it enters the sample holder. The optical gradient force is balanced symmetrically in the radial and longitudinal directions. However, the particle is drawn in the downstream direction of the laser beam by the scattering force. Even so, the particle is trapped stably because the optical gradient force along the laser axis is dominant over the scattering force in the case where the particle is much smaller than the wavelength of the laser light (Ashkin et al., 1986). The position of the trapped particle depends on laser power, particle size and refractive index of the particle. The trapped particle is thus positioned on the laser axis and slightly downstream of the focal point. The distance along the laser axis from the trap position of the particle to the focal point of the laser is a few hundred micrometres. Note that, owing to the wavelength of the laser light, the particle size, which is possible to manipulate in our optical trap, is almost up to $500 \mathrm{~nm}$.

As shown in Figs. 2(b) and 2(c), the sample cell, designed to be as small as possible to suppress air convection, is a rectangular-shaped cell $(8 \mathrm{~mm} \times 32 \mathrm{~mm} \times 12.5 \mathrm{~mm})$. A nonspherical lens is attached to one side of the sample cell to focus the laser light for the trap. A transparent thin film of polypropylene is attached to the opposite side to enable diffraction X-rays to pass through. The bottom surface of the sample cell is made of a thin film of stainless steel and the top surface is made of a thin glass plate with an anti-reflective coating. The sample cell is mounted on the $x y z$ positioning stages.

An ultrasonic transducer is installed under the sample cell, and sample particles are distributed in the cell by applying a short ultrasonic vibration pulse. Some particles wander into the trap region, where their kinetic energy is dissipated by viscous damping of air, and come to rest at the centre of the trap. Several minutes after the pulse is applied, all distributed particles, except one that is trapped, fall to the bottom of the sample cell.

The position of the trapped particle is monitored by two mutually orthogonal optical microscopes fitted with CCD video cameras. The top-view microscope is installed vertically so that it focuses on the sample position through the thin glass plate and is perpendicular to the OPS laser and the focused synchrotron radiation beam. The trapped sample particle is irradiated with the OPS laser, and the scattering light from the sample is monitored by the top-view microscope. The sideview microscope is installed horizontally so that it focuses on the sample position through the transparent thin film of polypropylene and is parallel to the OPS laser axis, i.e. perpendicular to the focused synchrotron radiation beam. A $532 \mathrm{~nm}$ notch filter is installed in front of the side-view microscope to avoid saturating the CCD video camera with the laser light. To observe the sample position with the sideview microscope, the sample is irradiated by a Gaussian beam with a power of about $4 \mathrm{~mW}$, which is produced by a semiconductor laser with a $780 \mathrm{~nm}$ wavelength. Using two optical microscopes with CCD video cameras makes it possible to monitor the position of the sample particle in three dimensions. The single-beam optical-trap sample holder is mounted on the $x y z$ positioning stages and aligned by the stages to keep the overlap between the sample position and the focal position of the synchrotron radiation during a diffraction measurement. However, it is impossible to take a clear image of a single particle of size less than $1 \mu \mathrm{m}$ with optical microscopes fitted with CCD video cameras. The particle looks larger than its true size because of the blooming effect of the CCD camera. As a result, it is difficult to adjust the overlap between the sample position and the focal position of the synchrotron radiation.

\subsection{X-ray diffractometer}

The X-ray diffractometer was installed and aligned at the SPring-8 BL40XU helical undulator beamline (Yasuda et al., 2009). The energy of the fundamental synchrotron radiation can be set between $8 \mathrm{keV}$ and $17 \mathrm{keV}$ by changing the undulator gap. A further monochromatic radiation beam $(\Delta E / E=$ $0.02 \%$ ) is formed by using an $\mathrm{Si}$ (111) channel-cut monochromator. To obtain sufficient photon flux density for measuring a single particle, the monochromatic synchrotron 
radiation beam is focused by a phase zone plate with a $300 \mathrm{~mm}$ focal length. Beam sizes of the focused synchrotron radiation are typically $3.0 \mu \mathrm{m}$ in the $x$ direction and $1.5 \mu \mathrm{m}$ in the $z$ direction. To exclude higher-order diffraction generated by the phase zone plate, an order-sorting aperture (OSA) was installed between the phase zone plate and the focal position. Photon flux density of the focused synchrotron radiation is

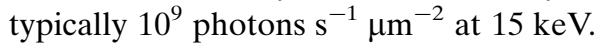

The single-beam optical-trap sample holder is aligned so that the synchrotron radiation beam crosses the axes of the OPS laser beam perpendicularly and the trapped sample is on the focal point of the synchrotron radiation beam by using the $x y z$ positioning stages. A Debye-Scherrer camera with a curved imaging plate (IP) is used to detect the weak X-ray diffraction pattern of the single particle. The camera length of the Debye-Scherrer camera is $286.48 \mathrm{~mm}$.

\section{X-ray diffraction measurement of a single $\mathrm{ZnO}$ particle}

$\mathrm{X}$-ray diffraction images of a single particle and an assemblage of $\mathrm{ZnO}$ particles were obtained. The particle was a standard reference material, $\mathrm{ZnO}$ (NIST standard reference material $674 \mathrm{~b}$ ), with a crystallite size of $201.4 \pm 2.5 \mathrm{~nm}$. It has a hexagonal (wurtzite) structure and space group $P 6_{3} m c$ with lattice parameters $a=3.249897 \pm 0.0000038 \AA$ and $c=5.20653$ $\pm 0.00035 \AA$. A scanning electron microscope (SEM) image of the assemblage of $\mathrm{ZnO}$ particles is shown in Fig. 3. Most of the $\mathrm{ZnO}$ particles are excellent in terms of crystallinity. The wavelength of the $\mathrm{X}$-ray synchrotron radiation was determined to be $\lambda=0.83231$ (7) $\AA$ by analysing the powder diffraction pattern of a silicon powder assemblage (NIST SRM 640c).

A single $\mathrm{ZnO}$ particle was levitated and trapped with the single-beam optical-trap sample holder by using the OPS laser set to $420 \mathrm{~mW}$. A photograph of the single $\mathrm{ZnO}$ particle suspended on the sample holder is shown in Fig. 4. The trapped particle wandered and rotated irregularly in the optical trap due to the Brownian motion of the background

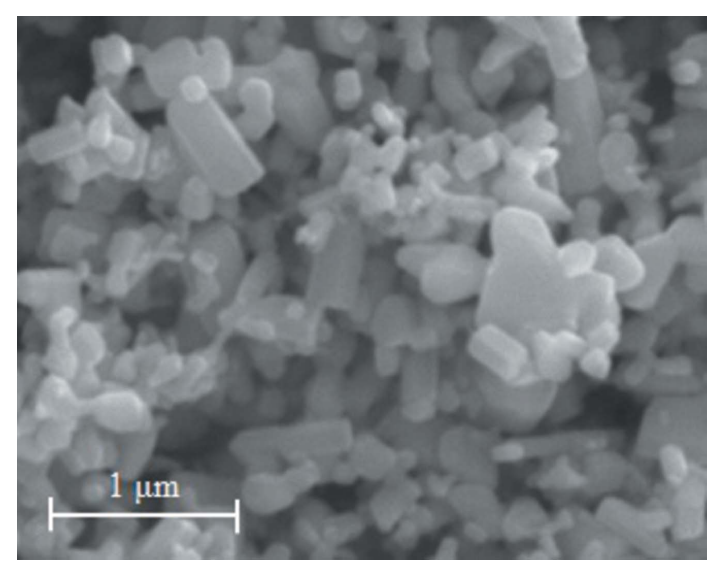

Figure 3

SEM image of an assemblage of ZnO particles (NIST SRM 674b).

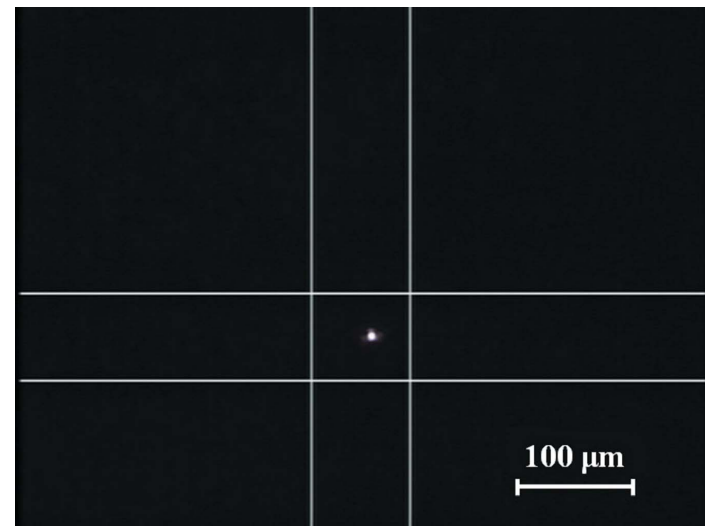

Figure 4

Photograph of a single $\mathrm{ZnO}$ particle taken by the side-view microscope. The four lines are markers synthesized on the monitor. The particle looks larger than its true size because of the blooming effect of the CCD camera.
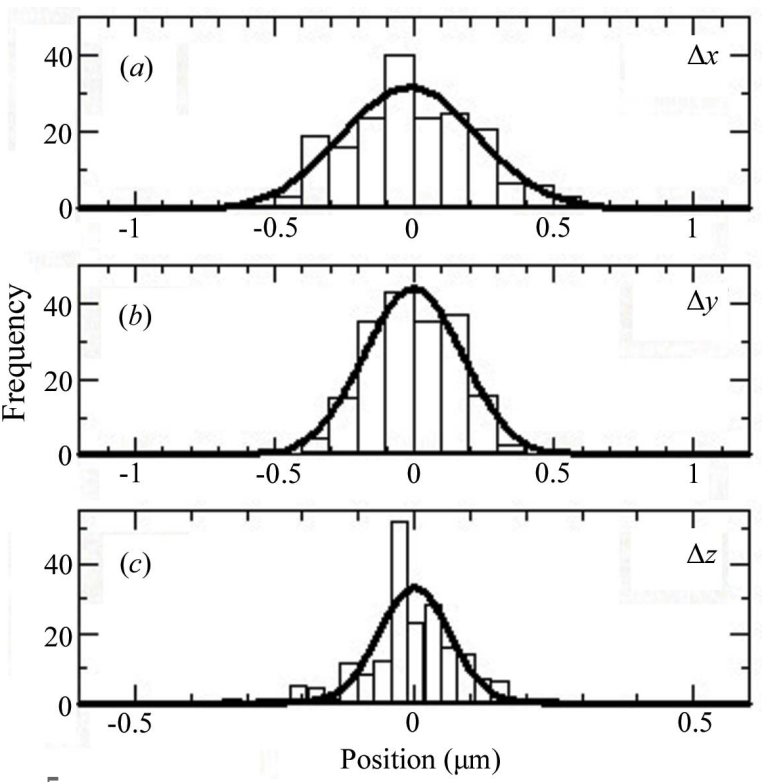

Figure 5

Histograms of deviation from the mean particle position in the (a) $x$ direction, $(b) y$ direction and $(c) z$ direction. The solid lines represent Gaussian curves. The best-fit values of the FWHM in the $x, y$ and $z$ directions are $0.56 \mu \mathrm{m}, 0.42 \mu \mathrm{m}$ and $0.15 \mu \mathrm{m}$, respectively.

air. Random motion of the trapped particle was recorded with the cameras attached to the top-view and side-view microscopes. Typical random motion was analysed over three minutes, and particle positions were determined every second. Histograms of the deviation from the mean particle position are shown in Figs. 5(a)-5(c). FWHMs of the distributions, defined as 'position jitter' in the $x, y$ and $z$ directions were $0.56 \mu \mathrm{m}, 0.42 \mu \mathrm{m}$ and $0.15 \mu \mathrm{m}$, respectively. Fig. $6(a)$ shows an $\mathrm{X}$-ray diffraction image of a single $\mathrm{ZnO}$ particle suspended with the single-beam optical-trap sample holder. The beam sizes of the focused synchrotron radiation were evaluated by knife-edge scanning using gold meshes. The beam sizes at the focal point were $3.01 \mu \mathrm{m}$ in the $x$ direction and $1.53 \mu \mathrm{m}$ in the $z$ direction. The photon flux density of the focused synchro- 
Table 1

Experimental conditions and results of X-ray diffraction measurements of $\mathrm{ZnO}$.

WH: Williamson-Hall method. Sch: Scherrer method.

\begin{tabular}{lll}
\hline & Single ZnO particle & Assemblage of ZnO particles \\
\hline X-ray beam size $(\mu \mathrm{m})$ & $3.01 \times 1.53$ & $100 \times 100$ \\
Photon flux density $\left(\right.$ photons s $\left.{ }^{-1} \mu \mathrm{m}^{-2}\right)$ & $2.82 \times 10^{9}$ & $6.51 \times 10^{6}$ \\
Irradiation time & $11.5 \mathrm{~h}$ & $180 \mathrm{~s}$ \\
Lattice parameters $(\AA)$ & $a=3.2505 \pm 0.0005, c=5.207 \pm 0.006$ & $a=3.24916 \pm 0.00002, c=5.20559 \pm 0.00004$ \\
Crystallite size $(\mathrm{WH})(\mathrm{nm})$ & $D=199.4 \pm 17.1$ & $D=193.5 \pm 16.3$ \\
Microstrain $(\mathrm{WH})(\mathrm{nm})$ & Negligible $\left(-7.0 \times 10^{-6} \pm 1.9 \times 10^{-5}\right)$ & Negligible $\left(-2.8 \times 10^{-5} \pm 1.8 \times 10^{-5}\right)$ \\
Crystallite size $\dagger(\mathrm{Sch})(\mathrm{nm})$ & $193.4 \pm 26.2$ & $193.1 \pm 13.9$ \\
\hline
\end{tabular}

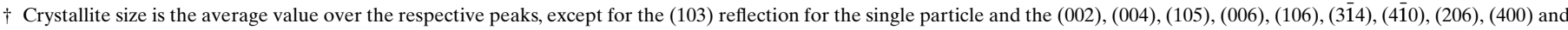
(401) reflections for the particle assemblage.

tron radiation was $2.82 \times 10^{9}$ photons s ${ }^{-1} \mu \mathrm{m}^{-2}$. The irradiation time of the focused synchrotron radiation was $11.5 \mathrm{~h}$. This irradiation time is not the accumulation time needed to randomize the diffraction pattern of the single particle. There was concern that the adjustment of the overlap between the sample position and the focal position of the synchrotron radiation might have been imperfect.

To evaluate the quality of the X-ray diffraction image of the single $\mathrm{ZnO}$ particle held in the single-beam optical trap, an $\mathrm{X}$-ray diffraction image of the assemblage of $\mathrm{ZnO}$ particles (NIST SRM 674b) packed in a glass capillary with $100 \mu \mathrm{m}$ inner diameter was also obtained, as shown in Fig. 6(b). The beam sizes of the parallel synchrotron radiation were $100 \mu \mathrm{m} \times 100 \mu \mathrm{m}$ in the $x$ and $z$ directions. The photon flux density of the parallel synchrotron radiation was $6.51 \times$ $10^{6}$ photons s${ }^{-1} \mu \mathrm{m}^{-2}$. The actual exposure time was $180 \mathrm{~s}$. The capillary was rotated at $2^{\circ} \mathrm{s}^{-1}$ during the measurement.

The diffraction image of the single $\mathrm{ZnO}$ particle shows a Debye ring pattern, which is similar to the powder diffraction pattern of the assemblage of $\mathrm{ZnO}$ particles. The Debye ring pattern can mainly be attributed to the irregular rotation of the particle. Unfortunately, the Debye ring pattern of the single $\mathrm{ZnO}$ particle has an azimuthally inhomogeneous intensity distribution.

The diffraction patterns of the single $\mathrm{ZnO}$ particle and the assemblage of $\mathrm{ZnO}$ particles were obtained by summing the

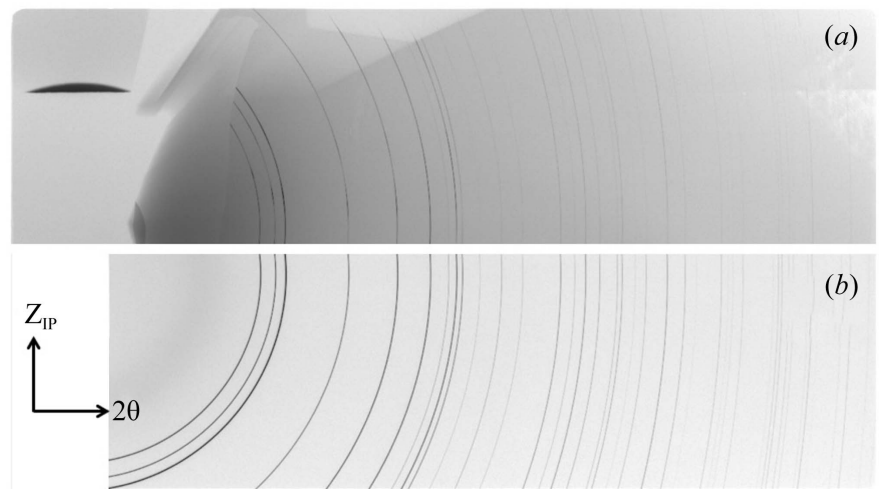

Figure 6

$\mathrm{X}$-ray diffraction images of $(a)$ the single $\mathrm{ZnO}$ particle and $(b)$ the assemblage of $\mathrm{ZnO}$ particles. signal intensities of 51 pixels $\left(50 \mu \mathrm{m}\right.$ pixel $\left.^{-1}\right)$ along the $z_{\mathrm{IP}}$ direction. The backgrounds of the diffraction patterns are subtracted by adjusting a polynomial. The intensities of the $\mathrm{X}$-ray diffraction are normalized to the intensity of the (101) reflection. The diffraction patterns of the single $\mathrm{ZnO}$ particle and the assemblage of $\mathrm{ZnO}$ particles are shown in Figs. 7(a) and $7(b)$.

As for the diffraction pattern of the single $\mathrm{ZnO}$ particle, because of the azimuthally inhomogeneous intensity distribution of the Debye ring pattern, it is difficult to apply the Rietveld method (Rietveld, 1967, 1969). Diffraction angles and widths of every reflection profile were determined by fitting the intensity data to a pseudo-Voigt function and by zero-point correction. The lattice parameters were determined to be $a=3.2505 \pm 0.0005 \AA$ and $c=5.207 \pm 0.006 \AA$ by using the diffraction angles of the trapped single particle and the plane spacing equation for the hexagonal structure. Experimental conditions and the results of the X-ray diffraction measurements of $\mathrm{ZnO}$ are summarized in Table 1. The relative diffraction intensity ratio for the single $\mathrm{ZnO}$ particle in the optical trap differed slightly from that for the assemblage; this difference can be attributed to insufficient random rotation of the single $\mathrm{ZnO}$ particle.

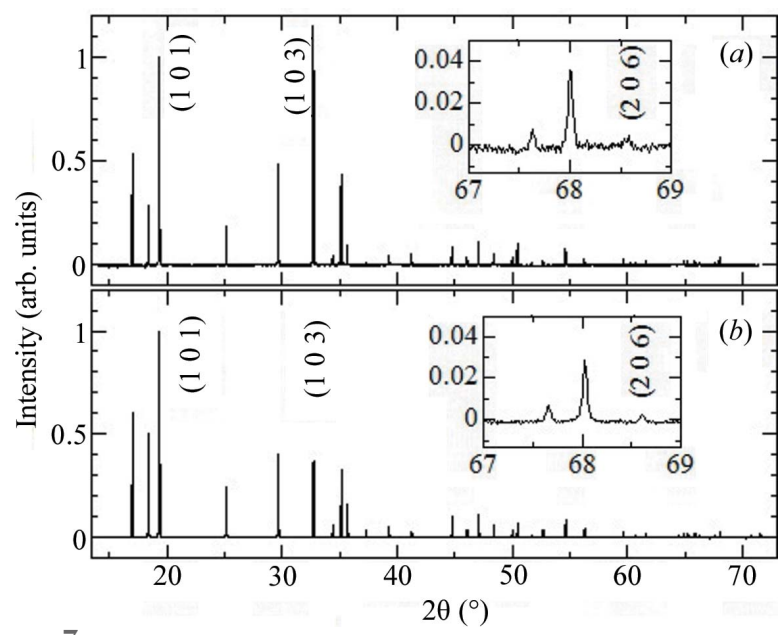

Figure 7

$\mathrm{X}$-ray diffraction profile of $(a)$ the single $\mathrm{ZnO}$ particle and $(b)$ the assemblage of $\mathrm{ZnO}$ particles. The insets in $(a)$ and $(b)$ emphasize the intensities around the (206) reflection. 
Table 2

Crystallite size $D_{h k l}$ along the respective reflection planes.

\begin{tabular}{|c|c|c|c|c|c|c|}
\hline \multicolumn{4}{|c|}{ Single $\mathrm{ZnO}$ particle } & \multicolumn{3}{|c|}{ Assemblage of $\mathrm{ZnO}$ particles } \\
\hline$h k l$ & $2 \theta\left(^{\circ}\right)$ & $\begin{array}{l}\text { Relative } \\
\text { intensity }\end{array}$ & $D_{h k l}(\mathrm{~nm})$ & $2 \theta\left({ }^{\circ}\right)$ & $\begin{array}{l}\text { Relative } \\
\text { intensity }\end{array}$ & $D_{h k l}(\mathrm{~nm})$ \\
\hline 100 & 17.018 & 0.534 & 177.571 & 17.026 & 0.602 & 172.137 \\
\hline 002 & 18.411 & 0.289 & 209.232 & 18.417 & 0.499 & $242.22 \dagger$ \\
\hline 101 & 19.364 & 1.000 & 198.082 & 19.372 & 1.000 & 161.446 \\
\hline 102 & 25.173 & 0.184 & 163.156 & 25.180 & 0.241 & 180.233 \\
\hline $2 \overline{1} 0$ & 29.690 & 0.482 & 170.921 & 29.703 & 0.403 & 186.695 \\
\hline 103 & 32.741 & 1.154 & $290.061 \dagger$ & 32.750 & 0.366 & 200.660 \\
\hline 200 & 34.417 & 0.042 & 184.270 & 34.430 & 0.060 & 185.295 \\
\hline $2 \overline{1} 2$ & 35.160 & 0.437 & 224.767 & 35.173 & 0.323 & 197.090 \\
\hline 201 & 35.694 & 0.090 & 158.505 & 35.708 & 0.160 & 185.297 \\
\hline 004 & 37.314 & 0.018 & 145.931 & 37.323 & 0.034 & $355.833 \dagger$ \\
\hline 202 & 39.303 & 0.044 & 154.336 & 39.319 & 0.051 & 189.996 \\
\hline 104 & 41.265 & 0.052 & 214.138 & 41.279 & 0.027 & 198.686 \\
\hline 203 & 44.771 & 0.089 & 213.591 & 44.786 & 0.103 & 190.574 \\
\hline $3 \overline{1} 0$ & 46.072 & 0.042 & 210.156 & 46.090 & 0.033 & 186.962 \\
\hline $3 \overline{1} 1$ & 47.083 & 0.115 & 206.161 & 47.100 & 0.107 & 192.369 \\
\hline $2 \overline{1} 4$ & 48.382 & 0.052 & 199.342 & 48.398 & 0.058 & 217.876 \\
\hline $3 \overline{1} 2$ & 50.013 & 0.038 & 227.870 & 50.031 & 0.036 & 181.348 \\
\hline 105 & 50.459 & 0.109 & 226.425 & 50.475 & 0.067 & $227.829 \dagger$ \\
\hline 204 & 51.648 & 0.013 & 153.968 & 51.666 & 0.010 & 171.953 \\
\hline 300 & 52.678 & 0.022 & 169.594 & 52.696 & 0.038 & 187.414 \\
\hline $3 \overline{1} 3$ & 54.633 & 0.082 & 199.379 & 54.653 & 0.083 & 197.875 \\
\hline 302 & 56.272 & 0.031 & 205.595 & 56.295 & 0.040 & 197.055 \\
\hline 006 & 57.333 & 0.004 & 242.035 & 57.345 & 0.006 & $317.415 \dagger$ \\
\hline 205 & 59.637 & 0.030 & 233.016 & 59.656 & 0.028 & 203.402 \\
\hline 106 & 60.258 & 0.010 & 154.157 & 60.276 & 0.007 & $297.369 \dagger$ \\
\hline $3 \overline{1} 4$ & 60.705 & 0.011 & 214.794 & 60.725 & 0.010 & $358.075 \dagger$ \\
\hline $4 \overline{2} 0$ & 61.628 & 0.021 & 182.787 & 61.652 & 0.018 & 209.313 \\
\hline $4 \overline{1} 0$ & 64.428 & 0.005 & 204.427 & 64.460 & 0.007 & $266.832 \dagger$ \\
\hline $4 \overline{2} 2$ & 64.903 & 0.015 & 187.015 & 64.928 & 0.021 & 196.441 \\
\hline $4 \overline{1} 1$ & 65.242 & 0.018 & 167.020 & 65.268 & 0.023 & 194.105 \\
\hline $2 \overline{1} 6$ & 65.872 & 0.022 & 203.909 & 65.891 & 0.018 & 220.262 \\
\hline 304 & 66.300 & 0.009 & 226.987 & 66.319 & 0.013 & 218.841 \\
\hline $4 \overline{1} 2$ & 67.638 & 0.010 & 182.701 & 67.666 & 0.008 & 188.495 \\
\hline $3 \overline{1} 5$ & 68.012 & 0.037 & 212.199 & 68.033 & 0.029 & 187.581 \\
\hline 206 & 68.577 & 0.005 & 169.841 & 68.613 & 0.004 & $289.457 \dagger$ \\
\hline 107 & 70.730 & 0.009 & 176.023 & 70.746 & 0.009 & 212.387 \\
\hline $4 \overline{1} 3$ & & & - & 71.573 & 0.022 & 187.637 \\
\hline 400 & & & - & 72.544 & 0.002 & $156.075 \dagger$ \\
\hline 401 & & & - & 73.313 & 0.006 & $236.465 \dagger$ \\
\hline
\end{tabular}

$\dagger$ Values of crystallite size $D_{h k l}$ were excluded from the calculation of crystallite size.

Reflection positions and their relative intensities for the single $\mathrm{ZnO}$ particle are listed in Table 2 . The intensity of the (101) reflection is most intense in the diffraction pattern of the assemblage of $\mathrm{ZnO}$ particles. As for the single $\mathrm{ZnO}$ particle, however, the intensity of the (103) reflection is stronger than that of the (101) reflection (see Fig. 7). It is conceivable that the enhancement of the (103) reflection is a result of the preferred orientation. However, the (206) reflection of the single $\mathrm{ZnO}$ particle is barely detectable, and the relative intensity of the (206) reflection is not enhanced. In addition, a single particle has various shapes. In the case of a rod-like crystal, the rotation around its own axis is enhanced frequently. Intensities of the reflections related to the planes parallel to the rotation axis can be enhanced. It is presumed that the strong intensity of the (103) reflection is attributed to the insufficient random rotation of the single particle and/or the morphology of the crystals; that is, it is not attributed to the preferred orientation. This presumption is consistent with the fact that the measured sample is a single $\mathrm{ZnO}$ particle.
As for the diffraction pattern of the assemblage of $\mathrm{ZnO}$ particles, the crystal structure was refined by the Rietveld method as implemented in TOPAS Academic (Version 6; Coelho, 2018). The assemblage of $\mathrm{ZnO}$ particles has a hexagonal (wurtzite) structure and space group $P 6_{3} m c$. The refined parameters included the scale factors, a background represented by a fifth-order Chebyshev polynomial with a $1 / x$ term, fundamental parameters for the instrument, lattice parameters, atomic coordinates and isotropic atomic displacement parameters. The lattice parameters were determined to be $a=3.24916 \pm 0.00002 \AA$ and $c=5.20559 \pm 0.00004 \AA$. The agreement factors for this Rietveld refinement are $R_{\mathrm{wp}}=$ $3.91 \%$ and $R_{\mathrm{p}}=1.98 \%$. It is presumed that the assemblage of $\mathrm{ZnO}$ particles has no preferred orientation because the agreement factors are not improved by the refinement of parameters for the preferred orientation.

\section{Crystallite size and microstrain}

Contributions of crystallite size and microstrain on diffraction peak shape broadening can be separated with the WilliamsonHall method (Williamson \& Hall, 1953). Broadening of the shape of the diffraction peak due to instrumental effects was obtained by analysing the powder diffraction pattern of a standard material such as silicon (with a crystallite size of $4.9 \mu \mathrm{m}$; NIST SRM 640c). The instrumental corrected broadening of the respective peaks $\left(\beta_{h k l}\right)$ was defined in terms of the FWHM of the diffraction peak. The relation between $\beta_{h k l}$, crystallite size $D(\mathrm{~nm})$ and microstrain $\varepsilon$ can be written as

$$
\beta_{h k l} \cos \theta=\frac{K \lambda}{D}+2 \varepsilon \sin \theta,
$$

where $K(K=0.8290)$ is Scherrer's constant, $\lambda$ is the wavelength of the $\mathrm{X}$-ray radiation and $\theta$ is the Bragg angle. Equation (1) is called the Williamson-Hall equation. A Williamson-Hall plot of the single $\mathrm{ZnO}$ particle is shown in Fig. 8. From fitting the objects to equation (1), crystallite size and microstrain were calculated to be $D=199.4 \pm 17.1 \mathrm{~nm}$ and $\varepsilon=-7.02 \times 10^{-6} \pm 1.85 \times 10^{-5}$, respectively (Table 1 ). Crystallite size and microstrain of the assemblage of $\mathrm{ZnO}$ particles packed in a glass capillary were also analysed by

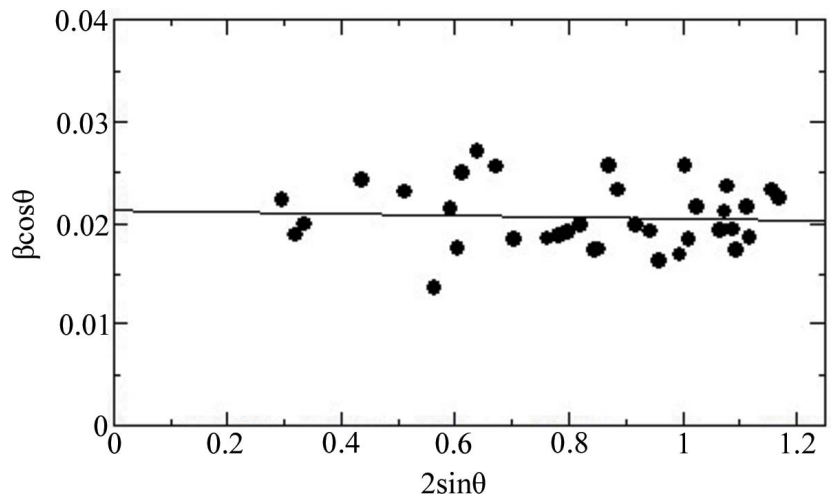

Figure 8

Williamson-Hall plot of the single $\mathrm{ZnO}$ particle. The solid line represents the fitting of the objects to the linear function given by equation (1). 
the Williamson-Hall method. Crystallite size and microstrain were calculated to be $D=193.5 \pm 16.3 \mathrm{~nm}$ and $\varepsilon=-2.80 \times$ $10^{-5} \pm 1.82 \times 10^{-5}$, respectively. It is concluded from these results that microstrain was negligible in both cases.

When microstrain is negligible, crystallite size $D_{h k l}$ along the respective reflection planes can be calculated with the Scherrer equation (Scherrer, 1918), namely

$$
\beta_{h k l}=\frac{K \lambda}{D_{h k l} \cos \theta} .
$$

$D_{h k l}$ of the single $\mathrm{ZnO}$ particle and that of the assemblage of $\mathrm{ZnO}$ particles packed in a glass capillary are listed in Table 2 and plotted in Fig. 9. As for the single $\mathrm{ZnO}$ particle, $D_{h k l}$ values for the respective diffraction peaks [except for the (103) reflection] are in the range $145-225 \mathrm{~nm}$. The value of $D_{103}$ is calculated to be $290.1 \mathrm{~nm}$. This value is prominent among the values of $D_{h k l}$ for the single $\mathrm{ZnO}$ particle. It is assumed that $D_{h k l}$ follows a normal distribution. To avoid the influence of the prominent value, the crystallite size of the single $\mathrm{ZnO}$ particle is defined as the average value over $D_{h k l}$ within $\mu \pm 2 \sigma$, where $\mu$ is the median and $\sigma$ is the square root of variance of the normal distribution. In particular, $D_{103}$ is excluded from the average. The crystallite size of the single $\mathrm{ZnO}$ particle is calculated to be $193.4 \pm 26.2 \mathrm{~nm}$, which is consistent with the result obtained by the Williamson-Hall method.

As for the assemblage of $\mathrm{ZnO}$ particles, there are several prominent values of $D_{h k l}$. To avoid the influence of these prominent values, the crystallite size is defined as the average value over $D_{h k l}$ within $\mu \pm 2 \sigma$. In particular, indexes of prominent values excluded from the average are (002), (004), (105), (006), (106), (314), (410), (206), (400) and (401). The crystallite size of the assemblage of $\mathrm{ZnO}$ particles is calculated to be $193.1 \pm 13.9 \mathrm{~nm}$, which is consistent with the result obtained by the Williamson-Hall method.

The distribution of $D_{h k l}$ for the single $\mathrm{ZnO}$ particle is wider than that for the assemblage of $\mathrm{ZnO}$ particles. Since the number of diffracted X-rays is proportional to the volume of the sample, the diffraction signal from the single $\mathrm{ZnO}$ particle is much weaker than that from the assemblage of $\mathrm{ZnO}$

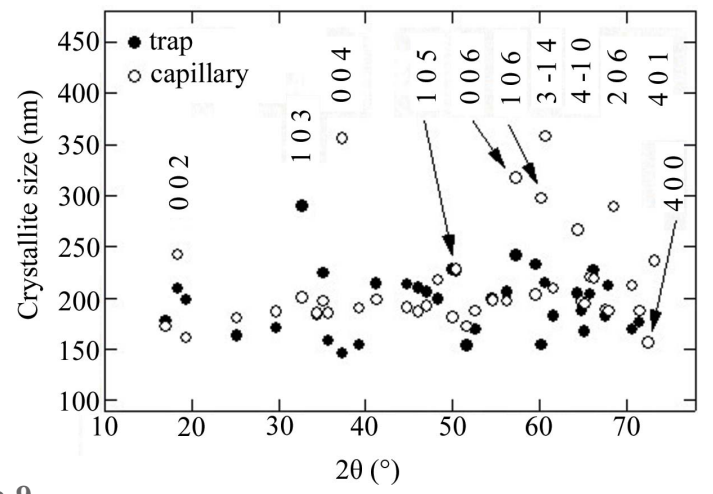

Figure 9

Crystallite size $\left(D_{h k l}\right)$ corresponding to each diffraction peak of the single $\mathrm{ZnO}$ particle (trap) and the particle assemblage (capillary). Indexes of the diffraction peaks where $D_{h k l}$ was calculated to be prominent are indicated in the figure. particles. The signal-to-noise ratio of the X-ray diffraction image for the single $\mathrm{ZnO}$ particle is lower than that for the assemblage of $\mathrm{ZnO}$ particles. The wide distribution of $D_{h k l}$ can be attributed to the low signal-to-noise ratio of the diffraction image.

\section{Discussion}

Recently, two-dimensional semiconductor photon-counting detectors have generally been used for X-ray diffraction measurement. Indeed, a photon-counting detector (DECTRIS, EIGER X 1M) was installed in the BL40XU beamline at SPring-8 to enable rapid diffraction measurements for single crystals of small molecules (Yasuda \& Kimura, 2019). This photon-counting detector has a sensitive area (width $\times$ height) of $77.2 \mathrm{~mm} \times 79.9 \mathrm{~mm}$ and pixel size of $75 \mu \mathrm{m} \times 75 \mu \mathrm{m}$. On the other hand, the IP has a sensitive area (width $\times$ height) of $400 \mathrm{~mm} \times 200 \mathrm{~mm}$ and readout resolution of $50 \mu \mathrm{m} \times 50 \mu \mathrm{m}$. To estimate crystallite size from diffraction peak broadening, it is necessary to obtain a diffraction profile with high spatial resolution. In addition, a wide range of diffraction angles $(2 \theta)$ is needed to evaluate the influence of preferred orientation and morphology of a crystal on crystallite size. In this work, the IP was used to achieve a wide sensitive area and high-spatial resolution simultaneously.

The laser power density with which the trapped particle was irradiated reached $10^{4} \mathrm{~W} \mathrm{~cm}^{-2}$. Under such intense laser irradiation, lattice expansion might be caused by the increased sample temperature. The heat release rate of the trapped particle into the environment is small because the trapped particle is being levitated in air without mechanical contact. Intense laser irradiation might also cause the charge configuration of the sample to change. In some cases, the change of charge configuration induces a slight change in the crystal structure. The X-ray diffraction image of the single $\mathrm{ZnO}$ particle held in the single-beam optical trap was compared with that of the assemblage of $\mathrm{ZnO}$ particles packed in a glass capillary. This comparison revealed no significant difference in lattice parameters. It is therefore concluded that the X-ray diffraction profile and crystal structure of the single $\mathrm{ZnO}$ particle are unaffected by intense laser irradiation.

In this work, the crystallite size of the single $\mathrm{ZnO}$ particle was successfully determined. It is not affected by the preferred orientation and size distribution of the assemblage of $\mathrm{ZnO}$ particles. Crystal structure and crystallite size of particles measured by traditional $\mathrm{X}$-ray diffraction were average values of the assemblage of $\mathrm{ZnO}$ particles with various crystallite sizes and shapes. Crystal structure and crystallite size of the single particle should be distinguished from those of the assemblage of $\mathrm{ZnO}$ particles. X-ray diffraction measurements of a single particle make it possible to investigate the one-toone relationship between crystal structure and crystallite size of a particle.

It should be noted that a single particle is not always a single grain. When a single particle is multi grain, the value of the crystallite size estimated from the diffraction peak broadening becomes an average value over the respective 
crystallite sizes of the particle. At present, it is difficult to extract the sample particle from the optical trap because it is still moving after the laser for the optical trap is stopped. It is important to know whether the measured single particle is a single grain or a multi grain. It is therefore necessary to develop a mechanism to extract a sample particle after a diffraction measurement.

It is well known that the actual value of Scherrer's constant $K$ for a particle assemblage depends on the crystallite size distribution (Langford \& Wilson, 1978). Further research on the crystallite size of a single particle and a particle assemblage would clarify the relation between Scherrer's constant $K$ and crystallite size distribution.

In air the trapped particle wandered and rotated irregularly in the optical trap due to the Brownian motion of the background air. It is difficult to stop and control such random motion and rotation because the viscous damping force by air is small. In this work, it was impossible to perform tomographic measurements because the size of the target particle was smaller than that of the focused synchrotron radiation beam. Even if the particle size is larger than that of the focused synchrotron radiation beam, the random motion and rotation of the particle will make it difficult to perform tomographic measurements for a single particle. In addition, the random motion and rotation decrease the actual exposure time of the X-ray radiation. However, an optical trap in an air environment has the advantage with regard to suppressing background scattering. Although motion and rotation of a single particle is stable and controllable in an optical trap with a liquid environment, a certain amount of background scattering cannot be avoided. The intensity of the X-ray diffraction signal is proportional to the volume of the particle. As a result, the signal-to-noise ratio of the $\mathrm{X}$-ray diffraction image degrades as the particle size decreases, especially in the size range lower than $100 \mathrm{~nm}$. Indeed, manipulation in an SEM (Burghammer, 1997) and an ultrathin sputtered adhesive layer or just a membrane sandwich made of graphene or $\mathrm{Si}_{3} \mathrm{~N}_{4}$ can be useful for the sample-holding method. These methods have mechanical contact. A single particle in the optical trap is isolated from other particles and walls. Since surface effects are enhanced in the case of nanometre-sized particles, X-ray diffraction measurements for a single particle of size less than $100 \mathrm{~nm}$ will make it possible to observe the physical effects of mechanical contact with other particles and walls. As for an optical trap under vacuum, the trapped particle is stable in motion because the motion of the particle is not affected by the Brownian motion of the background gas. In addition, there is no background scattering. To study size-dependent physical properties, an optical trap under vacuum is thus useful for $\mathrm{X}$-ray diffraction measurements for a single nanometre-sized particle.

\section{Conclusions}

A single-beam optical-trap sample holder for X-ray diffraction measurements of a single nanometre-sized particle was developed. A single $\mathrm{ZnO}$ particle was levitated and suspended in air with the optical gradient force of a focused laser beam without mechanical contact. An X-ray diffraction image of the single $\mathrm{ZnO}$ particle was obtained by combining the singlebeam optical-trap sample holder with focused synchrotron radiation. The diffraction image showed a Debye ring pattern, and the lattice parameters of the single $\mathrm{ZnO}$ particle are consistent with those of the assemblage of $\mathrm{ZnO}$ particles. The Debye ring diffraction pattern is thought to be mainly caused by the irregular rotation of the particle. The crystallite size of the single $\mathrm{ZnO}$ particle was determined by the Scherrer method to be $193.4 \pm 26.2 \mathrm{~nm}$.

\section{Funding information}

This work was partly supported by JASRI's GIGNO project and by a Grant-in-Aid for Scientific Research (C) (grant Nos. 25390012, 18K04904) from Japan's Ministry of Education, Culture, Sport, Science and Technology. This work was conducted at the BL40XU of SPring- 8 with the approval of the Japan Synchrotron Radiation Research Institute (proposal Nos. 2014B1373, 2015B1381, 2016A1364, 2017A1397, 2017B1480 and 2018B1316.

\section{References}

Aoyagi, S., Kuroiwa, Y., Sawada, A., Yamashita, I. \& Atake, T. (2002). J. Phys. Soc. Jpn, 71, 1218-1221.

Ashkin, A. (1970). Phys. Rev. Lett. 24, 156-159.

Ashkin, A., Dziedzic, J. M., Bjorkholm, J. E. \& Chu, S. (1986). Opt. Lett. 11, 288-290.

Burghammer, M. (1997). PhD thesis, Ludwig Maximilians University, Germany.

Coelho, A. A. (2018). J. Appl. Cryst. 51, 210-218.

Fukuyama, Y., Yasuda, N., Kimura, S. \& Takata, M. (2013). J. Phys. Soc. Jpn, 82, 114608-1-4.

Hoshina, T. (2013). J. Ceram. Soc. Jpn, 121, 156-161.

Inoue, K., Oka, T., Suzuki, T., Yagi, N., Takeshita, K., Goto, S. \& Ishikawa, T. (2001). Nucl. Instrum. Methods Phys. Res. A, 467-468, 674-677.

Kimura, S., Moritomo, Y., Tanaka, Y., Tanaka, H., Toriumi, K., Kato, K., Yasuda, N., Fukuyama, Y., Kim, J. E., Murayama, H. \& Takata, M. (2007). AIP Conf. Proc. 879, 1238-1241.

Langford, J. I. \& Wilson, A. J. C. (1978). J. Appl. Cryst. 11, 102-113.

Riekel, C., Burghammer, M. \& Schertler, G. (2005). Curr. Opin. Struct. Biol. 15, 556-562.

Rietveld, H. M. (1967). Acta Cryst. 22, 151-152.

Rietveld, H. M. (1969). J. Appl. Cryst. 2, 65-71.

Santucci, S. C., Cojoc, D., Amenitsch, H., Marmiroli, B., Sartori, B., Burghammer, M., Schoeder, S., DiCola, E., Reynolds, M. \& Riekel, C. (2011). Anal. Chem. 83, 4863-4870.

Scherrer, P. (1918). Nachr. Ges. Wiss. Gott. 2, 98-100.

Volkringer, C., Popov, D., Loiseau, T., Guillou, N., Ferey, G., Haouas, M., Taulelle, F., Mellot-Draznieks, C., Burghammer, M. \& Riekel, C. (2007). Nat. Mater. 6, 760-764.

Williamson, G. K. \& Hall, W. H. (1953). Acta Metall. 1, 22-31.

Yasuda, N. \& Kimura, S. (2019). AIP Conf. Proc. 2054, 050007.

Yasuda, N., Murayama, H., Fukuyama, Y., Kim, J. E., Kimura, S., Toriumi, K., Tanaka, Y., Moritomo, Y., Kuroiwa, Y., Kato, K., Tanaka, H. \& Takata, M. (2009). J. Synchrotron Rad. 16, 352-357. 